\title{
Évaluation de tests de dépistage des drogues sur le terrain
}

\section{Evaluation of roadside drug testing equipment}

Nele SAMYN*(1), Vincent ARESCHKA ${ }^{(1)}$, Alain VERSTRAETE ${ }^{(2)}$

(1) Institut National de Criminalistique et Criminologie, Bruxelles - BELGIQUE

(2) Laboratoire de Biologie Clinique-Toxicologie, Hôpital Universitaire, Gent - BELGIQUE

*Auteur à qui adresser la correspondance : Nele SAMYN, Institut National de Criminalistique et Criminologie, Chaussée de Vilvorde 100 - 1120 Bruxelles - BELGIQUE - Tél : +32 22400564 - Fax : +32 22416105

e-mail : Nele.Samyn@just.fgov.be

(Reçu le 8 février 2000 ; accepté le 2 mars 2000)

\section{$R E ́ S U M E ́$}

Dix-neuf tests rapides pour la détection de la drogue au volant ont été répertoriés dans la deuxième partie du projet Rosita. Ils sont commercialisés sur le marché international par au minimum 32 firmes. Seize tests ont été spécifiquement développés pour l'analyse de l'urine, trois pour la salive et un pour la sueur. Pour ce qui concerne l'urine, il y a au moins trois modalités pour l'analyser sur place : l'immersion d'une languette ou d'une carte, l'emploi d'une pipette, l'emploi d'un réservoir ad-hoc. L'utilisation de ces tests au laboratoire a permis d'en évaluer globalement la facilité d'utilisation sur le terrain. Une appréciation de "très bon" à "bon" a été attribuée à environ soixante pour-cent des tests et seulement un test a été jugé "inacceptable". Seuls les tests "Rapiscan" et "Drugwipe" peuvent utiliser un lecteur électronique de résultats, qui permet également un stockage aisé des résultats.

Ce travail a mis en évidence des difficultés concernant l'interprétation objective des résultats (absence de lecture électronique), des problèmes de détection et de spécificité dans le cas de l'ecstasy ainsi qu'un problème de spécificité dans le cas de la morphine (les autres opiacés sont également détectés). Une étude expérimentale dans laquelle sept tests rapides ont été comparés, a montré que les Syva Rapidtest, Rapid Drug Screen, Rapitest et Teststik étaient les plus fiables pour la détection des cannabinoïdes dans l'urine. Tous les tests, à l'exception du Frontline, étaient suffisamment sensibles pour la détection de la benzoylecgonine. Les résultats pour les opiacés étaient acceptables pour tous les tests. Syva Rapidtest, Rapid Drug Screen, Rapitest et Instatest ont montré de bons résultats pour la détection de l'amphétamine et de la MDMA; seul le Teststik et Testcup n'ont pas permis de détecter la MDMA, même à une concentration supérieure à $8000 \mathrm{ng} / \mathrm{ml}$. MOTS-CLÉS

Tests de dépistage, drogues au volant, stupéfiants, urine, salive, sueur.

\section{SUMMARY}

Nineteen different on-site drug testing devices have been documented in the second work package of the Rosita project, commercialised by 32 companies on the international market. Sixteen are designed for urine, three for saliva, one for sweat. For urine, there exist roughly three kinds of test designs to obtain a result with an on-site drug test: a "dip" test (teststrip or testcard), a "pipette" test (testcassette) and a "cup" test. Since most of the on-site devices were tested in the laboratory, an evaluation of their user friendliness was done by three members of the laboratory personnel. Approximately sixty percent of the evaluated devices had a users quotation of "good" to "very good", thirty percent was considered "acceptable", only one was "not acceptable". Only the Rapiscan saliva tester and the Drugwipe use an electronic reader, that also allows easy storage of data.

The objective interpretation of the result (absence of a reader), the detection of ecstasy and other designer amphetamines, and the specificity of the tests for the illicit amphetamines and morphine (other opiates are detected as well) are problem issues.

In an experimental study, Syva Rapidtest, Rapid Drug Screen, Rapitest and Teststik showed the best accuracy in the detection of cannabinoids in urine. All tests except Frontline showed sufficient sensitivity and specificity for the detection of benzoylecgonine in urine. All tests showed acceptable results for the detection of opiates in urine. Syva Rapidtest, Rapid Drug Screen, Rapitest and Instatest showed good results for the detection of amphetamine and MDMA in urine. Testcup and Teststik failed to detect MDMA, even at concentrations above $8000 \mathrm{ng} / \mathrm{ml}$.

\section{KEY-WORDS}

Screening devices, on-site testing, drugs of abuse, urine, sali$v a$, sweat. 


\section{Introduction}

Il existe un consensus dans la plupart des pays Européens concernant la nécessité de combattre l'usage de drogue au volant. Le problème de la conduite automobile sous l'influence de drogues a souvent été assimilé au fait que le conducteur d'un véhicule automobile doit toujours être à même de maîtriser son véhicule $(1,2)$. Il est le plus souvent difficile de mettre en évidence de manière formelle une capacité réduite, voire une incapacité, à maîtriser un véhicule automobile et de prouver ces faits objectivement au tribunal. En Allemagne, en Suède et en Belgique, pays ayant adopté une loi de "tolérance zéro" $(1,3)$, une suspicion initiale doit exister avant qu'une quelconque action judiciaire ne soit entreprise.

Une deuxième approche, complémentaire, consisterait à utiliser un test de terrain acceptable, susceptible de fournir à un officier de police une information supplémentaire sur une récente consommation de drogue, ceci tout comme pour la conduite en état d'intoxication alcoolique où des contrôles nécessitant une réponse quasi immédiate doivent être effectués. Le recueil de la salive et de la sueur est facile, peu invasif et le milieu collecté présente peu de risques d'adultération. En cas de consommation de stupéfiant, la salive est le liquide biologique dans lequel les concentrations sont les mieux corrélées aux concentrations sanguines. La sueur est considérée comme une alternative possible.

La seconde partie du projet Rosita (4) se pose comme objectif l'inventaire et une évaluation de l'état des connaissances en matière des tests de dépistage de terrain. Dix-neuf tests originaux existent sur le marché dont seize spécifiques aux urines. Ces tests sont commercialisés par approximativement 32 firmes présentes sur le marché international. Seulement trois tests sont spécifiques à la salive tandis qu'un de ces tests peut être utilisé pour analyser la sueur.

\section{Matériaux et Méthodes}

\section{Étude de Marché}

Le monde des tests de dépistage des drogues étant en pleine évolution, de nouveaux prototypes et de nouveaux fabricants voient régulièrement le jour. Dans un but d'évaluation et de comparaison, il était donc nécessaire de créer une base de données simple et clairement structurée de telle sorte que tout nouveau test puisse être aisément inventorié et que les paramètres de certains tests plus anciens ayant été modifiés puissent être revus.
Les caractéristiques principales des tests sont encodées dans un tableau standardisé tel qu'illustré par la Figure 1. Celles-ci sont issues entre autres d'un récent rapport sur l'exactitude et la facilité d'emploi des tests de terrain publié par le Dr. Dennis J. Crouch (Center for Human Toxicology, University of Utah, Salt Lake City), d'évaluations publiées dans diverses revues internationales (5-20), de posters présentés lors de congrès internationaux, de rapports internes fournis par les fabricants et distributeurs ainsi que de données disponibles sur le réseau Internet.

On observe que des tests quasi-similaires ou même identiques commercialisés par des firmes différentes, sont présents sur le marché sous différents noms (Tableau I). Les informations tirées du réseau Internet sont issues des différents sites des fabricants et distributeurs, de pages consacrées spécifiquement aux tests de dépistage sur le site du NCADI (National Clearinghouse for Alcohol and Drug Information) (21) et sur le site de la FDA (Food and Drug Administration) (22); il s'agit de sites où on peut se renseigner sur les tests ayant fait l'objet d'une étude.

Pour chaque classe de stupéfiant, la réactivité croisée des analytes possibles est présentée dans un rapport séparé. La réactivité croisée de l'analyte cible est considérée comme étant de $100 \%$ vu que le test doit être capable de détecter prioritairement la molécule mère dans la salive et la sueur, ce qui n'est pas le cas des tests spécifiques aux urines.

\section{Étude Expérimentale}

Le degré d'exactitude de sept tests de terrain pour la détection de la cocaine (COC), des opiacés (OPI), des cannabinoïdes (CAN) ainsi que des amphétamines/méthamphétamine et de la MDMA (AMP et/ou mAMP) a été évalué au cours d'une étude de laboratoire. Les tests suivants ont été étudiés suivant les instructions des producteurs : Testcup, 4 paramètres testés simultanément (AMP-CAN-COC-OPI), Teststik, un paramètre testé à la fois (AMP-CAN-COC-OPI) et Frontline, un paramètre testé à la fois (AMP-CANCOC-OPI) de Roche Diagnostics (Indianapolis, USA), Syva Rapidtest, 4 paramètres testés simultanément (mAMP-CAN-COC-OPI) + 1 paramètre testé isolément (AMP) de Dade Behring Inc. (San Jose, USA), Rapid Drug Screen (RDS), 5 paramètres testés simultanément (AMP-mAMP-CAN-COC-OPI) de American BioMedica Corp. (Hudson, USA), Rapitest, 5 paramètres testés simultanément (AMP-CAN-COCOPI-BZO) de Morwell Diagnostics (Suisse) et le One Step Instastrip, un paramètre testé à la fois (AMP- 
CAN-COC-OPI) de Cortez Diagnostics (Calabasas, USA).

Les urines, préalablement centrifugées, ont été stockées dans des tubes en polypropylène de $50 \mathrm{ml}$ et portées à la température ambiante avant analyse. Pour les tests Testcup, RDS et Rapitest, un volume d'urine assez important est nécessaire pour mener à bien le test. Seulement 60 tests Intrastrip ont été fournis pour l'évaluation. Les amphétamines, les cannabinoïdes, les métabolites de la cocaïne et les opiacés ont été recherchés par FPIA (Fluorescence polarization immuno assay; ADx, Abbott Diagnostics) dans toutes les urines. La sensibilité et la spécificité de cette technique est bien connue et évaluée. Les valeurs seuils employées sont les suivantes : $1000 \mathrm{ng} / \mathrm{ml}$ pour les amphétamines, $50 \mathrm{ng} / \mathrm{ml}$ pour les cannabinoïdes, $300 \mathrm{ng} / \mathrm{ml}$ pour la cocaïne et $300 \mathrm{ng} / \mathrm{ml}$ pour les opiacés.

Tous les résultats positifs issus de l'analyse par FPIA ont été confirmés par GC-MS ; les principaux analytes détectés ont été quantifiés à l'aide d'étalons internes deutérés. Les valeurs seuils pour les confirmations sont de : $500 \mathrm{ng} / \mathrm{ml}$ pour l'amphétamine, la méthamphétamine, la MDA, la MDMA, la MDEA et/ou le MBDB (les autres analogues des amphétamines ont été analysés qualitativement), $15 \mathrm{ng} / \mathrm{ml}$ pour le 11 -nor-d9-THC$\mathrm{COOH}, 150 \mathrm{ng} / \mathrm{ml}$ pour la benzoylecgonine et 300 $\mathrm{ng} / \mathrm{ml}$ pour la morphine, la codéine, la 6-acétylmorphi- ne (les autres opiacés ont été analysés qualitativement). Un grand nombre de résultats négatifs issus des analyses FPIA ont aussi été confirmés par GC-MS, spécialement en cas de discordance entre les résultats des tests de terrain et les résultats de l'analyse par FPIA.

Des résultats différents ont été obtenus avec chaque type de test pour une certaine classe de stupéfiants : de vrais positifs (VP), soit les résultats de tests de terrain positifs pouvant être confirmés par GC-MS, de vrais négatifs $(\mathrm{VN})$, soit les résultats de terrains négatifs pouvant être confirmés par FPIA et/ou par GC-MS, de faux positifs (FP), soit les résultats de terrain positifs ne pouvant être confirmés par GC-MS, de faux négatifs (FN), soit les résultats de terrains négatifs se révélant positif par GC-MS.

L'emploi de ces résultats permet de calculer certains paramètres importants tels que : la sensibilité (la capacité du test à pouvoir identifier comme positif un échantillon d'urine qui contient réellement l'analyte cible en concentration supérieure à la valeur seuil), la spécificité (la capacité d'un test à identifier comme négatif une urine dépourvue de l'analyte cible ou contenant une concentration inférieure à la valeur seuil), la valeur prédictive positive (VPP, la probabilité qu'un test positif correspond à un résultat effectivement positif), la valeur prédictive négative (VPN, la probabilité qu'un test négatif correspond à un résultat effectivement négatif).

Figure 1 : Les caractéristiques principales d'un test urinaire illustré à l'aide du tableau standardisé utilisé dans la seconde partie du projet Rosita.

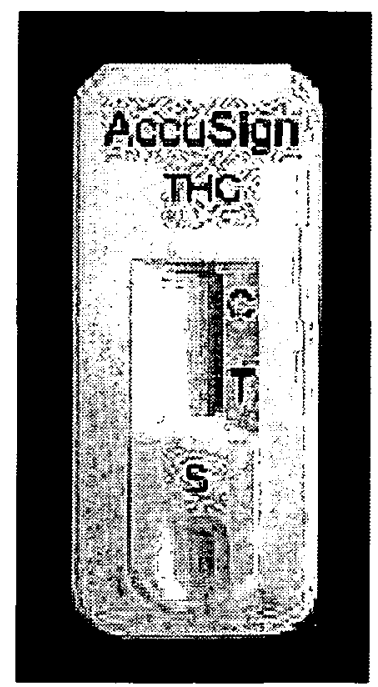

- Name of the original device : ACCUSIGN

- Manufacturer : Princeton BioMeditech

- Product identical to : Syva Rapidtest (Dade Behring)

Status DS (Lifesign L.L.C.)

Mahsan (Mahsan Diagnostika)

Dako (Veda Lab)

- Matrix :

- Available for :

- Number of parameters per device :

- Cost :

- FDA approved

- Storage conditions :

- Manipulations to obtain a result :

1) Aspirate urine with a plastic pipette (included in the pack)

2) Add 3 drops of sample to the sample well

3) Read the result in 3 - 5 minutes urine

AMP, mAMP, CAN, COC, OPI, PCP, BZO, BAR, TCA, MDN

$1-10$

10 - 15 EURO for a five panel

for single and multiple parameter tests

$2-30{ }^{\circ} \mathrm{C}$ 
positive/negative control line : present

Interpretation of the result : 1 line $=$ positive $/ 2$ lines $=$ negative possibility to store result : no

- Cut-off values :

\begin{tabular}{|c|c|}
\hline Cut-Off : & $\mathrm{ng} / \mathrm{m}$ \\
\hline amphetamine & 1000 \\
\hline barbiturates & 300 \\
\hline benzodiazepines & 300 \\
\hline cannabinoids & 50 \\
\hline benzoylecgonine & 300 \\
\hline methadone & 300 \\
\hline methamphetamine & 1000 \\
\hline morphine & 300 \\
\hline PCP & 25 \\
\hline tricyclics & 1000 \\
\hline
\end{tabular}

- User friendliness : good

- Available information :

ACCUSIGN

Verdonck I and Viaene B, Testing drugs of abuse with non-instrumental immunoassays: a field experience. Poster presented at the TIAFT/SOFT Joint Congress, October-November 1994, Tampa, Florida

Bogema SC, Performance study of four rapid on-site drug testing devices, presentation at AACC Conference Chicago 1998

Buchan BJ et al, J Forensic Sci, 43:395 (1998)

Kintz P and Giroud C, J Anal Toxicol, 21:589 (1997)

ACCUSIGN, SYVA RAPIDTEST

Hofbauer BM et al., Comparison of immunochromatographic rapid tests for screening of benzodiazepines, amphetamine and its derivatives (ecstasy) in urine. Poster presented at the TIAFT/SOFT Joint Congress, October 1998, Albuquerque, New Mexico

SYVA RAPIDTEST

Dade Behring: cross-reactivity testing of the Syva Rapidtest AMP and mAMP with MDA, MDMA, MDEA and MBDB

Mura P et al., Acta Clinica Belgica 1/99:35 (1999)

SYVA RAPIDTEST and STATUS:

An evaluation of Non-Instrumented Drug Test Devices. Report of the Substance Abuse and Mental Health Services Administration, Center for Substance Abuse Prevention,

Division of Workplace Programs. email: wvogl@samsha.gov

STATUS

Taylor HE et al., J Anal Toxicol 23:1 19 (1999)

- Comments and Conclusions :

- ADVANTAGES : - quick and inexpensive

- easy to use, no messy test after use

- a few drops of urine are sufficient to do the test properly, also when using a multi-panel device

- a large range of single tests and combinations are available

- built-in control line

- DISADVANTAGES : - Sometimes difficult to interpret the result: rather subjective (very faint line = negative?). The study of Verdonck et al. clearly shows that the \% of wrong results increases dramatically with non-trained operators. - PBM also manufacturers Syva Rapidtest and Status DS which are basically identical tests. This leads to confusion and difficulties to evaluate the reliability of the information provided by each distributor.

The results of the evaluation-studies of the different tests and cross-reactivities also show small differences.

\section{- ANALYTICAL CONCLUSIONS :}

Due to the contradictory results of the evaluation studies, one can conclude that the interpretation of the result is perhaps too subjective and certainly requires some experience.

Acceptable sensitivity for the designer amphetamines when combining both the AMP and mAMP test. More study is needed to interpret the "False Positives" possibly due to the presence of other amphetamine-like compounds. 
Annales de Toxicologie Analytique, vol. XII, $n^{\circ} 2,2000$

Tableau I : Résumé des tests de dépistage principaux, présentés dans la seconde partie du projet Rosita.

\begin{tabular}{|c|c|c|}
\hline Test original & Producteur & Tests identiques ou quasi-similaires \\
\hline Accusign & Princeton BioMeditech & $\begin{array}{l}\text { Syva Rapidtest (Dade Behring) } \\
\text { Status DS (Lifesign L.L.C.) } \\
\text { Mahsan (Mahsan Diagnostika) } \\
\text { Dako (Veda Lab) }\end{array}$ \\
\hline $\begin{array}{l}\text { Dip Drugscan-one } \\
\text { step }\end{array}$ & Syntron Bioresearch Inc. & $\begin{array}{l}\text { Quickscan, Quickpack II-one step, Quickstrip-one } \\
\text { step (Syntron) } \\
\text { One step Instastrip, Insta test, multiple drug screen } \\
\text { Instastrip, Insta test (Cortez Diagnostics) }\end{array}$ \\
\hline Drugcheck 5 & Drug Free Enterprises & $\begin{array}{l}\text { Drugstop (V-Tech, Inc.) } \\
\text { Teststrips = Dip Drug Scan-one test series (Syntron) }\end{array}$ \\
\hline Drugwipe & Securetec & Frontline test strips (Roche Diagnostics) \\
\hline DTx & Forefront Diagnostics & Instacheck \\
\hline First Check & Worldwide Medical Corp. & \\
\hline Frontline & Roche Diagnostics Corp. & \\
\hline Genie Cup & Point of Care Techn. & $\begin{array}{l}\text { Syva Rapid Cup d.a.u. } 5 \text { (Dade Behring) } \\
\text { Sure Step and Pharmscreen teststrips } \\
\text { (American Biomedical Corp.) }\end{array}$ \\
\hline Oral Screen & Avitar Technologies Inc. & Carepoint (Coventry, UK) \\
\hline $\begin{array}{l}\text { Pharmscreen Drug } \\
\text { Screen card }\end{array}$ & American Biomedical Inc. & $\begin{array}{l}\text { Dipro Drugscreen } 5 \text { panel (Dipro Diagnostic Products) } \\
\text { Surestep Drug Screen Card II (Biochem. } \\
\text { Immunosystems) } \\
\text { Clinistrip Drug Check Card (Clinicare Technologies) } \\
\text { Rapitest Multidrug Panel (Morwell Diagnostics) } \\
\text { Ultimed Surestick Drug Screen Card II (The Ultimate } \\
\text { Products) } \\
\text { Assurance Drug Screen Card (Applied Biotech) }\end{array}$ \\
\hline Quickscreen & PhamaTech & Surescreen (Surescreen Diagnostics) \\
\hline Rapid Drug Screen & AmericanBioMedica Corp. & \\
\hline Rapiscan & Cozart Bioscience Ltd. & \\
\hline Testcup & Roche Diagnostics Corp. & Teststik (Roche Diagnostics) \\
\hline Toxiquick & Bionike Laboratories Inc. & \\
\hline Triage & Biosite Diagnostics & \\
\hline Verdict II & Medtox Diagnostics Inc. & Profile II (Medtox) \\
\hline Visualine & Avitar Technologies Inc. & Sunline (Sun Biomedical Laboratories Inc.) \\
\hline
\end{tabular}




\section{Résultats et Discussion Étude de Marché}

Concernant les urines, il existe trois types de tests de terrain différents : un test nécessitant l'immersion partielle d'une languette ou d'une carte dans les urines durant quelques secondes (teststrip ou testcard), un test nécessitant l'emploi d'une pipette (testcassette ; quelques gouttes d'urine sont introduites dans le test à l'aide d'une pipette) et un test nécessitant l'emploi d'un réservoir ad-hoc (la partie immunoessai se trouvant dans le réservoir même). Certains exemples de tests urinaires sont présentés à la Figure 2. Le principe du dernier test mentionné ci-dessus semble intéressant, parce que le nombre de manipulations à effectuer est limité et que le prélèvement n'est jamais manipulée ; cependant, ce type de test nécessite un grand volume d'urine. Pour les tests nécessitant l'emploi d'une pipette, seules quelques gouttes d'urine sont nécessaires ; il existe des tests ne permettant l'analyse que d'un seul paramètre et des tests multiparamétriques (p. ex. Syva Rapidtest, Dade Behring ; Visualine, Avitar Technologies).

La plupart des tests sont disponibles pour la détection des amphétamines, de la méthamphétamine, des cannabinoïdes, de la cocaïne, des opiacés et de la phencyclidine (PCP). Soixante-dix pour-cent des tests disposent d'une détection séparée de l'AMP et de la mAMP. Quatre-vingt pour-cent des tests permettent la recherche des benzodiazépines (BZO) et des barbituriques, cinquante pour-cent permettent la recherche de la méthadone et seulement trente pour-cent permettent la recherche des antidépresseurs tricycliques.

Les tests peuvent être stockés à la température ambiante $\left(15-25^{\circ} \mathrm{C}\right)$. Les coûts des tests urinaires permettant l'analyse d'un paramètre unique varient de 2 à 6 Euro, et de 10 à 20 Euro pour les tests permettant l'analyse simultanée de 5 paramètres. Les tests multiparamétriques (1 à 5 paramètres) développés spécifiquement pour l'analyse de la salive coûtent de 6 à 18 Euro, tandis que le Rapiscan, qui utilise un lecteur de résultat électronique, est d'un coût plus élevé.

L'utilisation des tests de terrain par trois personnes expérimentées au laboratoire a permis d'en évaluer globalement la qualité d'emballage et d'étiquetage, la facilité d'utilisation et la facilité d'interprétation des résultats. Une appréciation "très bon" à "bon" a été attribuée à environ soixante pour-cent des tests ; trente pour-cent ont été jugés "acceptables" et seul le test Triage (Biosite) a été jugé inacceptable comme test de terrain.

Il y a plusieurs possibilités d'interpréter les résultats des tests : dans plus de quatre-vingt-dix pour-cent des cas, l'apparition d'une ligne à côté d'une ligne de contrôle indique un résultat négatif; l'intensité même de la ligne n'est pas importante. Une distinction claire entre un résultat positif ou négatif n'est pas toujours évidente surtout dans des conditions de luminosité faible ; un entraînement préalable avant de pouvoir
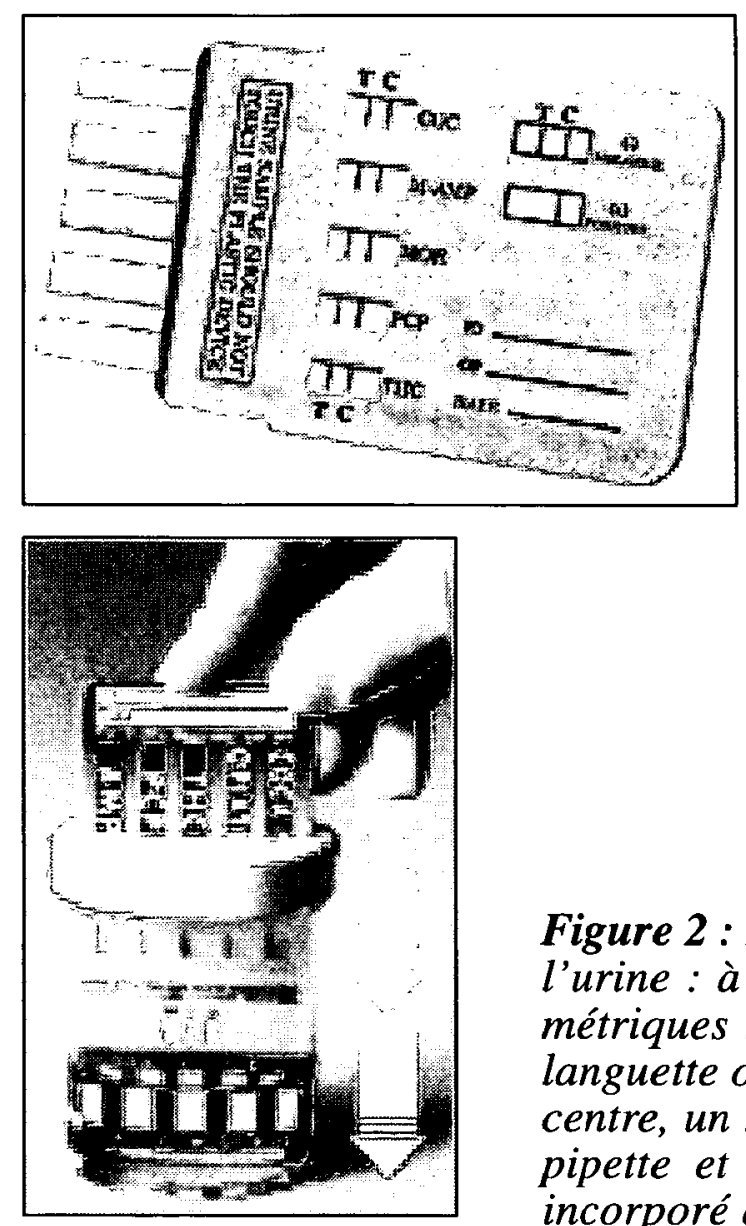

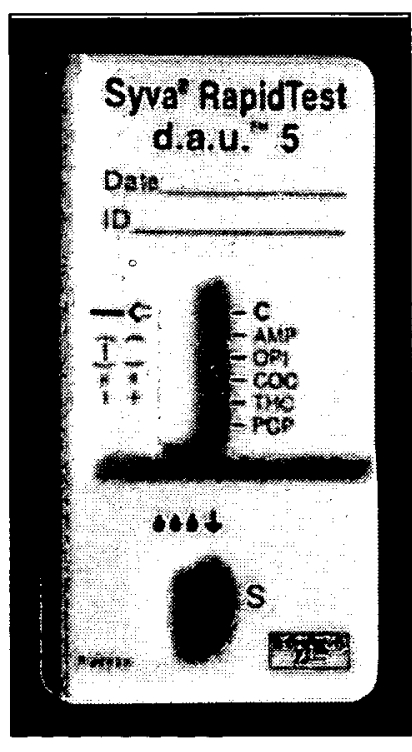

Figure 2 : Différents tests de terrain pour l'urine : à gauche, deux tests multiparamétriques nécessitant l'immersion d'une languette ou d'une carte dans l'urine, au centre, un test nécessitant l'emploi d'une pipette et à droite, un exemple de test incorporé dans un réservoir ad-hoc.

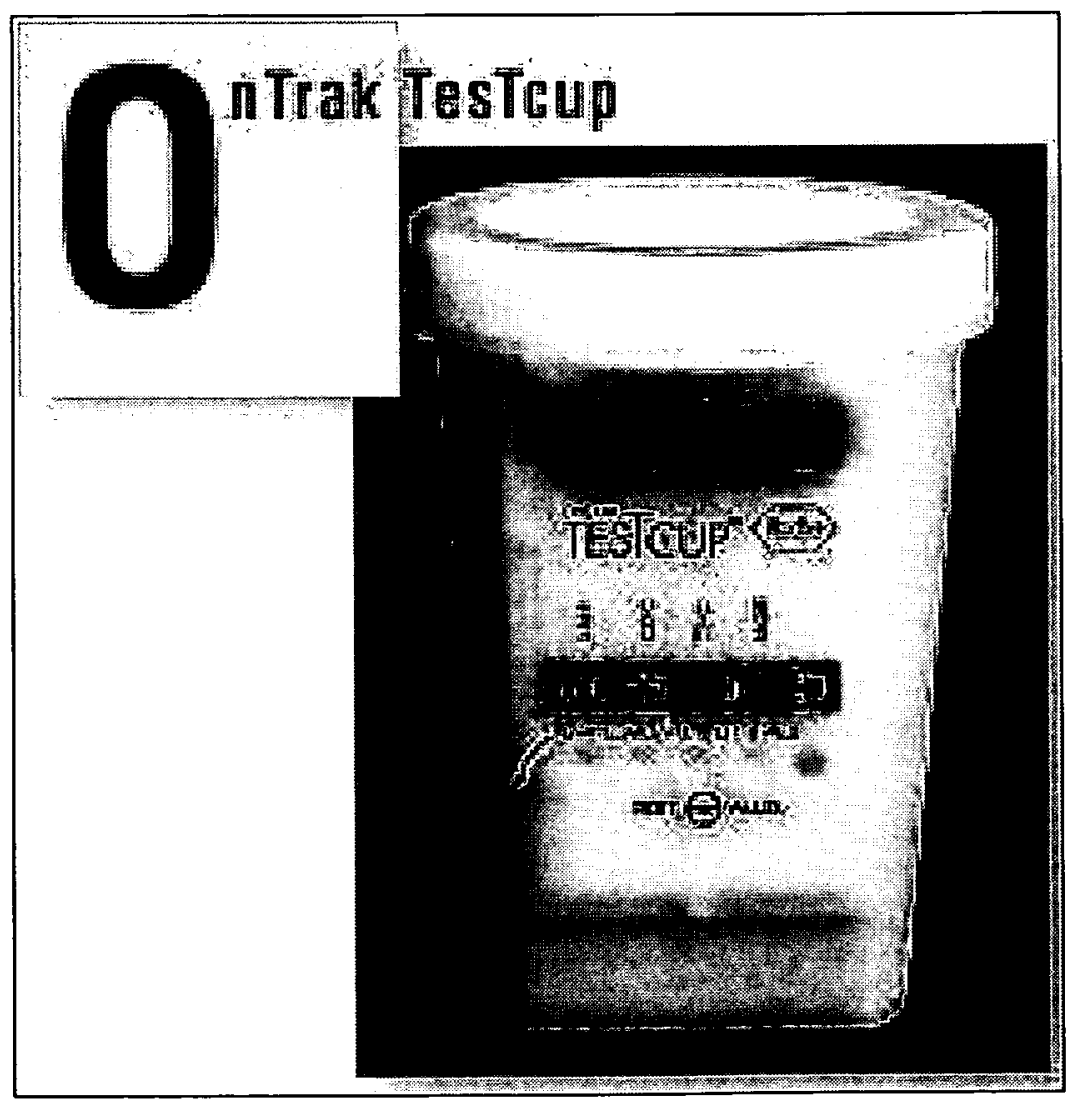


entreprendre les mêmes tests sur le terrain est donc nécessaire. Il est très important que le bon fonctionnement du test soit validé par une ligne de controle ; seuls les tests Frontline (Roche Diagnostics) et Drugwipe (Securetec $\mathrm{GmbH}$ ) ne disposent pas d'un tel contrôle. Le test Rapiscan (Cozart Bioscience Ltd., U.K.), spécifique à la salive, est le seul test disposant d'un lecteur de résultat électronique permettant un stockage aisé des données; un système de lecture du même type a été développé à l'état de prototype pour le test Drugwipe.

A de rares exceptions, les producteurs de tests de terrain spécifiques aux urines utilisent les valeurs seuil préconisées par le SAMHSA pour les drogues. L'utilisation de tests trop sensibles ou ayant des valeurs seuils s'écartant des valeurs théoriques augmente le nombre de faux positifs.

Dans bien des pays Européens, la consommation sans cesse croissante d'ecstasy implique l'emploi d'un test suffisamment sensible pour la MDMA et ses analogues. La réactivité croisée pour le test AMP est fixée à $100 \%$ pour la d-amphétamine; certains de ces tests montrent cependant une certaine réactivité croisée avec la MDA. La réactivité croisée de la MDA -entre autre métabolite de la MDMA- varie de 20 à $200 \%$. Les substances contenues dans certains décongestionnants nasaux et médicaments anorexiques (la b-phényléthylamine, la l-amphétamine, la phentermine, la phényl-propanolamine, la pseudoéphédrine et l'éphédrine) peuvent interférer de manière variable dans le test AMP. La réactivité croisée pour le test mAMP est fixée à $100 \%$ pour la d-méthamphétamine; les différents tests montrent cependant une réactivité croisée de 14 à $100 \%$ avec la MDMA. Très peu de données concernant d'autres molécules de la famille de l'ecstasy sont disponibles. La d-amphétamine, la l-méthamphétamine et certaines substances médicamenteuses peuvent interférer avec le test mAMP.

Tous les tests spécifiques aux opiacés montrent une réactivité croisée élevée avec un certain nombre de substances : la morphine et le 3-glucuronide de morphine, les métabolites de l'héroïne, la codéine ainsi que d'autres antitussifs, des antagonistes et des agonistes morphiniques. Une identification non ambiguë par GCMS de la molécule ayant induit un test positif pour les opiacés est donc dans tous les cas indispensable.

En conclusion, les problèmes principaux des tests de terrain sont la difficulté d'interprétation des résultats (absence de lecture électronique), la détection de l'ecstasy et d'autres molécules de la même famille ainsi que la spécificité des tests pour les amphétamines et la morphine.

\section{Étude Expérimentale}

Les performances des tests ont été évaluées en terme de leurs "Valeur Prédictive Positive" et de leurs "Valeur Prédictive Négative" ainsi qu'en terme de pourcentage de faux positifs et de faux négatifs. Il s'agit de paramètres qui représentent la probabilité d'obtenir un résultat positif ou négatif correct ; un pourcentage élevé, ou un VPP élevé indiquent qu'il existe une probabilité élevée qu'un résultat positif issu d'un test puisse être confirmé comme positif ou comme négatif pour un VPN élevé. La combinaison de tous les résultats corrects positifs ou négatifs représente une estimation de l'exactitude globale en terme de résultats positifs et négatifs pour chaque test étudié. Le test hypothétique idéal ne donne pas de faux positifs ni de faux négatifs et a un VPP et un VPN de 100.

Le nombre d'échantillons présentant une concentration en analyte proche de la valeur seuil préétablie pour la confirmation, a aussi été pris en compte comme critère d'évaluation des tests de terrain; la classification "positive" ou "négative" de ces échantillons est parfois théorique et les résultats de ces tests de terrain sont moins importants par rapport aux échantillons clairement positifs ou négatifs.

Certains tests peuvent identifier un pourcentage élevé d'échantillons positifs tout en donnant un grand nombre de faux positifs ; ces tests ont les caractéristiques d'un 'test agressif". Par opposition, certains tests donnent peu de faux positifs mais n'identifient pas un nombre élevé de vrais positifs ; ces tests ont les caractéristiques d'un "test conservateur".

Le Tableau II présente les résultats issus de tests de terrains pour la détection du 11-nor-d9-THC-COOH dans les urines. Les tests Syva Rapidtest, Rapid Drug Screen et Rapitest présentent le niveau de fiabilité le plus élevé. Des quatre FP obtenus avec le Teststik, trois sont issus de l'analyse d'échantillons ayant une concentration proche de la valeur seuil ; le Teststik est donc un test fiable. Huit FP ont été obtenus avec le Testcup, deux issus d'échantillons ayant une concentration proche de la valeur seuil et deux issus d'échantillons ayant respectivement une concentration de 7 et $8 \mathrm{ng} / \mathrm{ml}$ de 11 -nor-d9-THC-COOH, ce qui équivaut à 4 vrais FP. Ces résultats sont en accord avec l'étude de Buchan et al. (5) qui rapporte un très faible pourcentage de FP dans les échantillons d'urine prélevés chez des conducteurs suspects de conduite automobile sous l'influence des médicaments et stupéfiants. Le FN issu du Testcup a été obtenu en analysant un échantillon 
d'urine contenant $41 \mathrm{ng} / \mathrm{ml}$ de 11-nor-d9-THC-COOH. Le Teststik donne quant-à lui un résultat positif pour la même urine. Les différences obtenues entre ces deux tests sont difficilement explicables; il est probable qu'elles puissent être attribuées à des problèmes de manipulation du Testcup. Les tests Frontline et Instatest fournissent trop de faux négatifs pour des échantillons d'urines ayant une concentration supérieure à la valeur seuil et ne peuvent donc être jugés fiables pour la détection des cannabinoïdes dans les urines; de plus l'interprétation des résultats de ces tests est difficile. Mura et al. (13) ont obtenu de bons résultats pour la détection des cannabinoïdes dans l'urine avec le Syva Rapidtest, le test Dako (test identique), le test Biomedix et l'Instatest. Le test Frontline a fourni de nombreux résultats faussement négatifs.

Tableau II : Évaluation de l'exactitude des tests de terrain de détection des cannabinoüdes dans l'urine.

\begin{tabular}{|c|c|c|c|c|c|c|}
\hline & n & VP & VN & FP & FN & $(+,+)$ \\
\hline TESTCUP & 69 & 36 & 24 & 8 & 1 & 2 \\
\hline TESTSTIK & 87 & 48 & 35 & 4 & 0 & 3 \\
\hline FRONTLINE & 87 & 40 & 37 & 2 & 8 & 2 \\
\hline RAPIDTEST & 87 & 46 & 39 & 0 & 2 & 2 \\
\hline RDS & 60 & 31 & 26 & 3 & 0 & 2 \\
\hline RAPITEST & 56 & 30 & 23 & 3 & 0 & 1 \\
\hline INSTASTRIP & 59 & 25 & 24 & 1 & 9 & 2 \\
\hline
\end{tabular}

*FN ou FP issus de l'analyse d'échantillons ayant une concentration de 11-nor-d9-THC-COOH proche de la valeur seuil $(15 \mathrm{ng} / \mathrm{ml})$.

Le Tableau III présente les résultats de la détection de la benzoylecgonine dans les urines. Tous les tests, à l'exception du test Frontline, sont suffisamment spécifiques et sensibles pour détecter ce métabolite de la cocaïne dans les urines. Des deux FP obtenus avec le Testcup, le premier est issu de l'analyse d'un échantillon ayant une concentration proche de la valeur seuil, le second d'un échantillon contenant $106 \mathrm{ng} / \mathrm{ml} \mathrm{de}$ benzoylecgonine. Des deux FN obtenus avec le Teststik, le premier est issu de l'analyse d'un échantillon ayant une concentration proche de la valeur seuil, le second d'un échantillon contenant $313 \mathrm{ng} / \mathrm{ml}$ de benzoylecgonine. Le test RDS a fourni un résultat négatif pour un échantillon nettement positif par ailleurs confirmé par GC-MS.
Tableau III : Évaluation de l'exactitude des tests de terrain de détection de la cocä̈ne dans l'urine.

\begin{tabular}{|c|c|c|c|c|c|c|}
\hline & $\mathbf{n}$ & VP & VN & FP & FN & $(t /)^{*}$ \\
\hline TESTCUP & 69 & 18 & 49 & 2 & 0 & 1 \\
\hline TESTSTIK & 85 & 17 & 66 & 0 & 2 & 1 \\
\hline FRONTLINE & 86 & 14 & 66 & 1 & 5 & 1 \\
\hline RAPIDTEST & 86 & 19 & 67 & 0 & 0 & 0 \\
\hline RDS & 60 & 15 & 43 & 0 & 2 & 1 \\
\hline RAPITEST & 56 & 15 & 41 & 0 & 0 & 0 \\
\hline INSTASTRIP & 59 & 9 & 49 & 0 & 1 & 1 \\
\hline
\end{tabular}

*FN ou FP issus de l'analyse d'échantillons ayant une concentration de benzoylecgonine proche de la valeur seuil $(150 \mathrm{ng} / \mathrm{ml})$.

Le Tableau IV présente les résultats des test de terrain pour la détection des opiacés dans l'urine. Le test $R D S$ se distingue par sa fiabilité. Tous les autres tests fournissent des résultats "acceptables" pour la détection des opiacés. Des trois FP obtenus avec le Syva Rapidtest, deux sont issus d'échantillons contenant respectivement 74 et $120 \mathrm{ng} / \mathrm{ml}$ de morphine ; le dernier a aussi fourni un résultat positif avec le test Instastrip. Bien que l'analyse par FPIA soit positive et que l'analyse par GC-MS ait confirmé la présence de codéine en concentration élevée dans une urine, trois tests effectués sur cette même urine ont fourni un résultat négatif, deux tests ont fourni un résultat douteux et seuls les tests RDS et Testcup mais non le Teststik ont fourni un résultat positif. Cette urine était très foncée.

Tableau IV : Évaluation de l'exactitude des tests de terrain de détection des opiacés dans l'urine.

\begin{tabular}{|c|c|c|c|c|c|c|}
\hline & n & VP & VN & FP & FN & $(+/)^{*}$ \\
\hline TESTCUP & 69 & 15 & 53 & 1 & 0 & 0 \\
\hline TESTSTIK & 82 & 16 & 65 & 0 & 1 & 0 \\
\hline FRONTLINE & 86 & 14 & 68 & 0 & 4 & 0 \\
\hline RAPIDTEST & 86 & 16 & 65 & 3 & 2 & 0 \\
\hline RDS & 60 & 14 & 46 & 0 & 0 & 0 \\
\hline RAPITEST & 56 & 10 & 44 & 1 & 1 & 0 \\
\hline INSTASTRIP & 59 & 17 & 39 & 2 & 1 & 0 \\
\hline
\end{tabular}

*FN ou FP issus de l'analyse d'échantillons ayant une concentration d'opiacés proche de la valeur seuil $(300 \mathrm{ng} / \mathrm{ml})$. 
Le Tableau $\mathbf{V}$ présente les résultats pour la détection des amphétamines et/ou de la MDMA dans les urines. Les résultats de ces tests sont considérés comme positifs quand le paramètre AMP et/ou mAMP, s'il est disponible, montre un résultat positif. Les tests Syva Rapidtest (Amp et mAMP), RDS (AMP et $m A M P$ ), Rapitest ( $A M P$ ) et Instatest (AMP) fournissent de bons résultats pour la détection des amphétamines et de la MDMA dans les urines. Parmi les six FN obtenus avec le test RDS, trois proviennent de l'analyse d'échantillons ayant une concentration proche de la valeur seuil et trois des quatre $\mathrm{FN}$ avec le Rapitest sont similaires. Il en va de même pour deux des trois FN issus du test Syva Rapidtest ; les six FP issus de ce test n'ont pas pu être confirmés par GC-MS ou par FPIA. La présence d'autres substances apparentées aux amphétamines ne donnant pas lieu à une réaction croisée par FPIA ne peut cependant être complètement exclue. Une étude précédente, dans laquelle un grand nombre de tests rapides incluant l'AMP et la mAMP avaient été évalués, avait déjà mis en évidence une sensibilité acceptable pour la détection de l'ecstasy et d'autres molécules similaires dans l'urine par le Syva Rapidtest, Accusign, Instatest, Frontline, Triage et Toxiquick [20]. Les tests Testcup et Teststik fournissent des résultats très cohérents, ces deux tests ne détectent pas la présence de la MDMA, même à des concentrations supérieures à $8000 \mathrm{ng} / \mathrm{ml}$ alors que l'amphétamine est détectée avec une bonne sensibilité.

L'exactitude des tests a été résumé par la moyenne d'une cote analytique prenant en compte les paramètres de performance mentionnés ci-dessus. Ces résultats sont présentés dans le Tableau VI. Les résultats obtenus pour le Testcup et Teststik sont en accord avec les évaluations

Tableau V : Évaluation de l'exactitude des tests de terrain de détection de l'amphétamine et/ou de la MDMA dans l'urine.

\begin{tabular}{|c|c|c|c|c|c|c|c|}
\hline & n & VP & VN & FP & FN & MDMA & $(+/-)^{*}$ \\
\hline TESTCUP & 68 & 12 & 46 & 0 & 10 & 10 & 3 \\
\hline TESTSTIK & 85 & 16 & 56 & 0 & 13 & 11 & 3 \\
\hline FRONTLINE & 85 & 25 & 48 & 8 & 4 & 11 & 1 \\
\hline RAPIDTEST & 85 & 26 & 50 & 6 & 3 & 11 & 2 \\
\hline RDS & 59 & 15 & 38 & 0 & 6 & 9 & 3 \\
\hline RAPITEST & 55 & 16 & 35 & 0 & 4 & 8 & 3 \\
\hline INSTATEST & 58 & 28 & 28 & 1 & 1 & 11 & 1 \\
\hline
\end{tabular}

*FN ou FP issus de l'analyse d'échantillons ayant une concentration d'amphétamine ou de MDMA proche de la valeur seuil $(500 \mathrm{ng} / \mathrm{ml})$. des études précédemment publiées $(5,7,16,17,20)$. Une étude a bien évalué en détail le Rapitest (12) mais pas le test multiple. Le test simple pour les cannabinoïdes, la cocaïne, les opiacés et la méthamphétamine montre aussi une très bonne exactitude par rapport à l'analyse par GC-MS. Une valeur seuil faible signalée pour le RDS et qui avait donné lieu à plusieurs FP n'a pas été confirmée dans notre étude. Ce ne fut pas le cas non plus de l'excellente sensibilité du test Frontline pour détecter les cannabinö̈des, la benzoylecgonine et les opiacés dans les échantillons d'urine provenant des départements de médecine clinique et légale (18). L'interprétation des résultats est subjective car la lecture est effectuée par comparaison avec une échelle de couleurs. La distinction entre beige clair, rose pâle ou foncé n'est pas aisée : c'est pourquoi l'utilisation du test Frontline au bord de la route est déconseillée.

\section{Évaluation des tests pour la salive et la sueur}

Quelques exemples de tests spécifiques à la salive sont présentés dans la Figure 3.

\section{Facilité d'emploi}

Le test Drugwipe (Securetec $\mathrm{GmbH}$ ) est le seul test ne nécessitant pas une prise préalable de la salive ; ce test peut être aussi employé pour l'analyse de la sueur. L'absence d'un ligne de contrôle et d'une version permettant l'analyse simultanée de plusieurs paramètres est cependant un inconvénient majeur ; ce test est très facile à utiliser. Un lecteur de résultat est disponible pour en améliorer la fiabilité.

Le test Oral Screen Morphine (Avitar Technologies) se compose d'un outil de recueil spécifique à la salive et d'une testcassette classique comme pour l'urine.

Le Rapiscan (Cozart Bioscience Ltd.) est la combinaison d'un système de prélèvement de la salive et d'un lecteur électronique. L'innovation de ce dernier consiste dans la capacité de détecter plusieurs classes de drogues dans un même échantillon de salive. 
Tableau VI : Cote analytique des tests.

\begin{tabular}{|ccccc|}
\hline & CANNABIS & COCAINE & OPIACES & AMPMMMA \\
\hline TESTCUP & ++ & +++ & ++ & - \\
TESTSTIK & +++ & +++ & +++ & - \\
FRONTLINE & - & - & + & + \\
RAPIDTEST & +++ & +++ & ++ & ++ \\
RDS & +++ & ++ & +++ & ++ \\
RAPITEST & +++ & +++ & +++ & +++ \\
INSTATEST & - & +++ & +++ & +++ \\
\hline
\end{tabular}

$-=$ pas acceptable

$+=$ acceptable

$++=$ bon

$+++=$ très bon
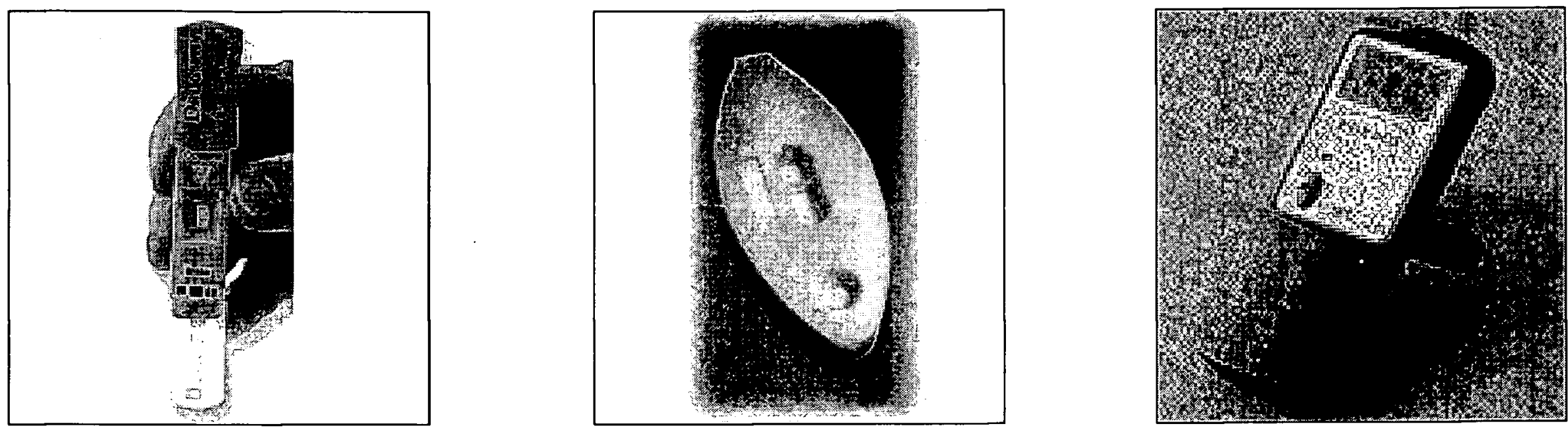

Figure 3: Tests étudiés pour le dépistage de la salive : à gauche, le test Drugwipe, au centre, le test Oral Screen Morphine et à droite, le test Rapiscan.

\section{Valeurs seuils et réactivité croisée}

Aucune valeur seuil officielle n'a été recommandée pour le dépistage des drogues dans la salive et la sueur. Le choix d'une valeur seuil pour un analyte donné est liée à sa concentration dans la salive et la sueur et à la sensibilité de la technique analytique utilisée. Deux données ne peuvent être négligées : (1) la salive et la sueur contiennent généralement les drogues parents alors que les urines contiennent leurs métabolites ; (2) les concentrations dans la salive sont beaucoup plus basses que dans l'urine. Cette première donnée est liée à la spécificité du test alors que la seconde est liée à la sensibilité.

Le test Drugwipe fournit des résultats acceptables pour les amphétamines, la cocaïne et les opiacés $(9,14)$. Le test Oral Screen présente une sensibilité suffisante pour détecter de faibles concentrations de morphine. Le test Rapiscan donne lieu à une réaction croisée importante avec l'amphétamine et la morphine et acceptable avec la cocaïne, mais manque de sensibilité pour la MDMA (ecstasy) et le THC, la substance active du cannabis.

\section{Remerciements}

Nous tenons à remercier plus particulièrement Michael Walsh pour les renseignements précieux qu'il nous a fourni, les fabricants ayant mis leur tests à notre disposition, Dr. Pascal Kintz pour les échantillons mis à notre disposition ainsi que Bart Viaene, Leen Vandevenne et Marina Puddu pour leur aide pratique. Ce travail a été partiellement financé par le DG 7 de la Commission Européenne, contrat DG VII PL98-3032. 


\section{Références}

1. Krüger H.-P., Perrine B.M.W., Mettke M., Huessy F Road traffic and illicit drugs. Overview of the legal provisions, difficulties faced by the police, and analysis of prevention attempts in selected European countries. In: P-PG/Circrout (98) 4, Pompidou Group, Council of Europe. Strasbourg, 1999.

2. Moeller M., Steinmeyer S., Aberl F. Operational, user and legal requirements across EU member states for roadside drug testing equipment. ROSITA Contract DG VII PL98-3032.

3. Charlier C., Verstraete A., Plomteux G. La législation dans les différents pays européens. In: Mura P., ed. Alcool, médicaments, stupéfiants et conduite automobile. Paris : Elsevier, 1999 ; 215-32.

4. http : //www.rosita.org

5. Buchan B.J., Walsh J.M., Leaverton P.E. Evaluation of the accuracy of on-site multi-analyte drug testing devices in the determination of the prevalence of illicit drugs in drivers. J. Forensic Sci. 1998 ; 43 : 395-99.

6. Buechler K.F., Moi S., Noar B., McGrath D., Villela J., Clancy M., Shenhav A., Colleymore A., Valkirs G., Lee T., Bruni J.F., Walsh M., Hoffman R., Ahmuty F., Nowakowski M., Buechler J., Mitchell M., Boyd D., Stiso N., Anderson R. Simultaneous detection of seven drugs of abuse by the Triage panel for drugs of abuse. Clin. Chem. $1992 ; 38: 1678-84$.

7. Crouch D.J., Frank J.F., Farrell L.J., Karsch H.M., Klaunig J.E. A multiple-site laboratory evaluation of three on-site urinalysis drug-testing devices. J. Anal. Toxicol. $1998 ; 22: 493-502$.

8. Fitzgerald R.L., Rexin D.A., Herold D.A. Detecting benzodiazepines: immunoassays compared with negative chemical ionization gas chromatography/ mass spectrometry. Clin. Chem. 1994 ; 40 : 373-80.

9. Kintz P., Cirimele V., Ludes B. Codeine testing in sweat and saliva with the Drugwipe. Int. J. Legal Med. 1998 ; $111: 82-4$

10. Kintz P., Giroud C. Immunoassay responses of MBDB. J. Anal. Toxicol. $1997 ; 21$ : 589-90.

11. Koch T.R., Raglin R.L., Scheree K., Bruni J.F. Improved screening for benzodiazepine metabolites in urine using the Triage panel for drugs of abuse. J. Anal. Toxicol. $1994 ; 18: 168-72$.
12. Korte T., Pykäläinen J., Lillsunde P., Seppälä T. Comparison of Rapitest with EMIT d.a.u. and GC-MS for the analysis of drugs in urine. J. Anal. Toxicol. $1997 ; 21: 49-53$.

13. Mura P., Kintz P., Papet Y., Ruetsch G., Piriou A. Evaluation de 6 tests rapides pour le dépistage du cannabis dans la sueur, la salive et les urines : corrélation avec la GC/MS. Acta Clinica Belgica 1999 ; Sup1/99 : 35-8.

14. Samyn N., van Haeren C. On-site testing of saliva and sweat with Drugwipe and determination of concentrations of drugs of abuse in saliva, plasma and urine of suspected users. Int. J. Legal Med. 2000 (in press).

15. Schwartz J.G., Hurd I.L., Carnahan J.J. Determination of tricyclic anti-depressants for ED analysis. American Journal of Emergency medicine 1994 ; 12 : 513-16.

16. Taylor E.H., Oertli E.H., Wolfgang J.W. Accuracy of five on-site immunoassay drugs-of-abuse testing devices. J. Anal. Toxicol. $1999 ; 23: 119-24$

17. Towt J., Tsai J., Hernandez M.R., Klimov A.D., Kravec C.V., Rouse S.L., Subuhi H.S., Twarowska B., Salamone S.J. Ontrak Testcup : a novel, on-site, multi-analyte screen for the detection of abused drugs. J. Anal. Toxicol. $1995 ; 19$ : 504-10.

18. Wennig R., Moeller M.R., Haguenoer J.M., Marocchi A., Zoppi F., Smith B.L., de la Torre R., Carstensen C.A., Goerlach-Graw A., Schaeffler J., Leinberger R. Development and evaluation of immunochromatographic rapid tests for screening of cannabinoids, cocaine, and opiates in urine. J. Anal. Toxicol. $1998 ; 22: 148-55$.

19. Wu A.H.B., Wong S.S., Johnson K.G., Callies J., Shu D.X., Dunn W.E., Wong H.Y. Evaluation of the Triage system for emergency Drugs-of-abuse testing in urine. J. Anal. Toxicol. $1993 ; 17: 241-45$.

20. Hofbauer B.M., von Meyer L.K. Comparison of immunochromatographic rapid tests for screening of benzodiazepines, amphetamine and its derivatives (ecstasy) in urine. In the abstract book of the TIAFT/SOFT Joint Congress, October 1998, Albuquerque, USA.

21.http://www.health.org/workplce/summary.htm

22.http://www.fda.gov/scripts/cdrh/cfdocs/cfpmn/search.cfm 